\title{
Lentiviral Vector Gene Transfer of Endostatin/Angiostatin for Macular Degeneration (GEM) Study
}

\author{
Peter A. Campochiaro, ${ }^{1, *}$ Andreas K. Lauer, ${ }^{2}$ Elliott H. Sohn, ${ }^{3}$ Tahreem A. Mir, \\ Stuart Naylor, Matthew C. Anderton, Michelle Kelleher, Richard Harrop, \\ Scott Ellis, and Kyriacos A. Mitrophanous ${ }^{5}$ \\ ${ }^{1}$ The Wilmer Eye Institute, Johns Hopkins University School of Medicine, Baltimore, Maryland; ${ }^{2}$ Casey Eye Institute, Oregon Health and Science University, Portland \\ Oregon; ${ }^{3}$ Department of Ophthalmology, University of lowa Hospitals and Clinics, lowa City, lowa; ${ }^{4}$ Formerly of Oxford BioMedica (UK) Ltd., Oxford, United Kingdom; \\ ${ }^{5}$ Oxford BioMedica (UK) Ltd., Windrush Court, Oxford, United Kingdom.
}

Neovascular age-related macular degeneration (NVAMD) is a prevalent cause of vision loss. Intraocular injections of VEGF-neutralizing proteins provide benefit, but many patients require frequent injections for a prolonged period. Benefits are often lost over time due to lapses in treatment. New treatments that sustain anti-angiogenic activity are needed. This study tested the safety and expression profile of a lentiviral Equine Infectious Anemia Virus (EIAV) vector expressing endostatin and angiostatin (RetinoStat $\left.{ }^{\circledR}\right)$. Patients with advanced NVAMD were enrolled at three centers in the United States, and the study eye received a subretinal injection of $2.4 \times 10^{4}(n=3), 2.4 \times 10^{5}(n=3)$, or $8.0 \times 10^{5}$ transduction units (TU; $n=15$ ). Each of the doses was well-tolerated with no dose-limiting toxicities. There was little or no ocular inflammation. There was one procedure-related serious adverse event (AE), a macular hole, which was managed without difficulty and resolved. There was a vector dose-related increase in aqueous humor levels of endostatin and angiostatin with high reproducibility among subjects within cohorts. Mean levels of endostatin and angiostatin peaked between 12 and 24 weeks after injection of $2.4 \times 10^{5}$ TU or $8.0 \times 10^{5}$ TU at $57-$ $81 \mathrm{ng} / \mathrm{mL}$ for endostatin and $15-27 \mathrm{ng} / \mathrm{mL}$ for angiostatin, and remained stable through the last measurement at week 48. Long-term follow-up demonstrated expression was maintained at last measurement (2.5 years in eight subjects and $>4$ years in two subjects). Despite an apparent reduction in fluorescein angiographic leakage that broadly correlated with the expression levels in the majority of patients, only one subject showed convincing evidence of anti-permeability activity in these late-stage patients. There was no significant change in mean lesion size in subjects injected with $8.0 \times 10^{5} \mathrm{TU}$. These data demonstrate that EIAV vectors provide a safe platform with robust and sustained transgene expression for ocular gene therapy.

Keywords: age-related macular degeneration (AMD), equine infectious anemia viral (EIAV) vector, lentiviral vector, subretinal injection, ocular gene therapy, neovascularization

\section{INTRODUCTION}

AGE-RELATED MACULAR DEGENERATION (AMD) is a highly prevalent cause of vision loss that is characterized by deposits called drusen and diffuse thickening of Bruch's membrane beneath the retinal pigment epithelium (RPE). As drusen become larger and merge, the microenvironment becomes less supportive, compromising function and survival, leading to loss of RPE cells and the overlying photoreceptors, which are dependent upon RPE cells for survival. ${ }^{1}$ The death of RPE and photo- receptors is slow, and the rate can vary greatly among patients, but eventually it can lead to patches of atrophy that correspond to blank spots in the central visual field, which over time can spread into the fovea and compromise visual acuity. ${ }^{2} \mathrm{Su}-$ perimposed on this slow neurodegenerative disease process is another disease process in which neovascularization (NV) occurs beneath the RPE and retina. The subgroup of AMD patients with this complication are said to have neovascular AMD (NVAMD). The risk of developing NVAMD varies

${ }^{*}$ Correspondence: Dr. Peter A. Campochiaro, Maumenee 815, Johns Hopkins University School of Medicine, 600 N. Wolfe Street, Baltimore, MD 21287-9277. E-mail: pcampo@jhmi.edu 
with age, ethnicity, life-style, severity of drusen, and pigmentary changes in the macula. In the Age Related Eye Disease Study, the oldest participants with the most extensive drusen and pigmentary changes had a $48 \%$ risk of developing NVAMD over 10 years. ${ }^{3}$ Compared with the overall group of patients with AMD, those with NVAMD experience relatively rapid reduction in visual acuity due to leakage of plasma from incompetent new vessels and collection of fluid in the neural retina, which compromises the function of photoreceptors and second-order neurons. The loss of vision is reversible if the excess fluid is eliminated in a timely fashion, but permanent loss of central vision necessary for reading and driving can occur from chronic persistent/recurrent fluid and/or subretinal fibrosis due to recruitment of other cells by the endothelial cells of the subretinal NV. Intraocular injections of a vascular endothelial growth factor (VEGF)-neutralizing protein can reduce leakage of plasma from subretinal NV, allowing excess fluid to be pumped out of the neural retina, resulting in improvement in visual acuity. ${ }^{4}$ However, in most patients with NVAMD, the excess production of VEGF is chronic and requires repeated injections of VEGF-neutralizing proteins to prevent recurrent and eventually permanent loss of vision.

The proangiogenic and pro-permeability effects of VEGF are countered by other proteins that are generated during the process of normal wound healing to help terminate physiologic NV when wound repair is completed. Some of these antiangiogenic proteins, such as pigment epitheliumderived factor (PEDF), ${ }^{5}$ are produced by nonvascular cells involved in wound repair. Others are generated by proteolytic cleavage of proteins with other functions, including endostatin, ${ }^{6}$ a cleavage product of collagen XVIII, and angiostatin, ${ }^{7}$ a cleavage product of plasminogen. Since these proteins are the normal "brakes" for physiologic NV, it is reasonable to hypothesize that increasing their expression could help to terminate and control pathologic NV. In mouse models of subretinal NV, which predicted the benefit of VEGF-neutralizing proteins in NVAMD, ${ }^{8,9}$ increased expression of PEDF,${ }^{10,11}$ endostatin, ${ }^{12,13}$ or angiostatin ${ }^{14}$ suppressed subretinal NV. Proof-of-concept for this approach was provided by a clinical trial in which intraocular injection of an adenoviral vector expressing PEDF showed evidence of decrease in leakage and reduction of intraretinal and subretinal fluid and blood in patients with NVAMD. ${ }^{15}$ However, the short duration of expression achieved with adenoviral vectors is not ideal for a chronic disease.
Lentiviral vectors are useful for gene transfer in the nervous system because they transduce dividing and non-dividing cells, including neurons ${ }^{16-20}$ and photoreceptors ${ }^{21-23}$; they elicit minimal inflammatory response that could compromise the viability of transduced cells; they have a large capacity of $10 \mathrm{~kb}$; and they integrate into the chromosomes of transduced cells, enabling stable long-term transgene expression. ${ }^{24}$ Equine Infectious Anemia Virus (EIAV) is a lentivirus that causes a chronic but non-fatal infection in horses but does not cause disease in humans. The EIAV-based lentiviral vector has all EIAV sequences deleted, except essential cis elements so that no EIAV proteins are expressed, and contains the woodchuck posttranscriptional regulatory element for enhanced expression. ${ }^{25,26}$ This vector is also under development for the treatment of Parkinson's disease, and a Phase I/II clinical trial has been carried out in 15 patients. ${ }^{27}$ Long-term, high-level of expression of green fluorescent protein (GFP) occurred in RPE cells underlying the small retinal detachment caused by the subretinal injection of EIAV.GFP in mice. ${ }^{28}$ Subretinal injection of EIAV vectors containing murine endostatin or angiostatin or a bicistronic version (RetinoStat ${ }^{\circledR}$ ) suppressed subretinal NV and reduced associated leakage. ${ }^{29,30}$ Herein the results of a Phase I clinical trial testing the safety of subretinal injection of RetinoStat are reported (Supplementary Fig. S1; Supplementary Data are available online at www.liebertpub.com/ hum) in which a cytomegalovirus promoter drives expression of human endostatin and angiostatin in patients with advanced NVAMD.

\section{MATERIALS AND METHODS}

The Gene transfer of Endostatin/angiostatin for Macular degeneration (GEM) study was an open-label, dose-escalation Phase I study at three clinical sites in the United States (Johns Hopkins University Hospital, Baltimore, MD; Oregon Health and Science University, Portland, OR; and University of Iowa Hospitals and Clinics, Iowa City, IA). Institutional Review Board approval was obtained at each site, and each subject provided informed consent. The study complied with the Declaration of Helsinki and the Health Insurance Portability and Accessibility Act, and was registered at www .clinicaltrials.gov under the identifier NCT01301443. Twenty-one subjects with advanced NVAMD were enrolled in three dose-ranging cohorts $\left(2.4 \times 10^{4}\right.$ transduction units [TU], $2.4 \times 10^{5} \mathrm{TU}$, and $8.0 \times 10^{5}$ $\mathrm{TU} ; n=3$ for each) and one cohort at the maximum tolerated dose (MTD; $\left.8.0 \times 10^{5} \mathrm{TU} ; n=12\right)$. The 
duration of the study was 48 weeks after which the subjects were encouraged to enroll in a 15-year long-term follow-up study.

\section{Study objectives and endpoints}

The primary objective of this study was to evaluate the safety and tolerability of a single subretinal injection of RetinoStat in patients with advanced NVAMD by monitoring the incidence of adverse events (AEs), the change in best-corrected visual acuity (BCVA) measured by Early Treatment Diabetic Retinopathy Study protocol, ${ }^{31}$ the magnitude and duration of ocular inflammation, and changes in intraocular pressure (IOP). Vector biodistribution (into blood and urine) and immune responses against vector constituents (detected in serum or aqueous humor tap samples) were also measured. Serum samples prepared from blood samples from each patient 4 weeks before surgery and 2, 4, 12,24 , and 48 weeks after treatment were analyzed by semi-quantitative enzyme-linked immunosorbent assay (ELISA) for antibody responses against RetinoStat components (including vector particle, transgene, and packaging cell line proteins). Biodistribution of RetinoStat to blood cells was assessed by analysis of DNA extracted from buffy coat samples with a real-time quantitative polymerase chain reaction (PCR)-based assay. Vector shedding was assessed by analysis of RNA extracted from urine.

An important secondary objective was to assess transgene expression by measuring levels of endostatin and angiostatin in aqueous humor at baseline and at multiple time points after subretinal injection of RetinoStat. Another secondary objective was to assess anti-permeability and antiangiogenic activity by measuring (1) changes in central subfield thickness (CST) using spectral domain-optical coherence tomography (SD-OCT; CST is the average of 21 measurements on six scans within the central $1 \mathrm{~mm}$ of the macula [total of 126 measurements], and is a quantitative measure of macular thickening that occurs from intraretinal or subretinal fluid used in all interventional clinical trials in subjects with NVAMD); (2) lesion size by image analysis of fluorescein angiograms; (3) change in leakage on fluorescein angiograms; and (4) changes in BCVA.

\section{Subject eligibility and exclusion criteria}

Patients with advanced NVAMD who showed evidence of leakage by fluorescein angiography (FA) and intraretinal/subretinal fluid by SD-OCT were eligible for the dose-escalation phase (cohorts $1-3$ ) if the study eye had BCVA $\leq 20 / 200$ (with better vision in the fellow eye), a poor visual prog- nosis due to subretinal fibrosis beneath the fovea or other evidence of foveal damage, and the patient was no longer a candidate for or refused standard care. Patients with advanced NVAMD were eligible for the MTD cohort (cohort 4) if they had BCVA $\leq 20 / 80$ and less severe fibrosis.

\section{Viral vector}

A single lot of RetinoStat was manufactured by a triple transient transfection of HEK293T cells as previously described. ${ }^{32}$ The vector was purified and concentrated by anion exchange chromatography and hollow-fiber ultrafiltration. A schematic of the RetinoStat genome is shown in Supplementary Fig. S1.

\section{Subretinal injection of RetinoStat}

Subjects were brought to the operating room, and under local anesthesia and sedation, or in some cases general anesthesia (depending upon surgeon and patient preference), vitrectomy was performed on the study eye. If the posterior hyaloid was still attached, it was separated from the retina over the optic nerve, dissected to the periphery, cut, and aspirated. A site for subretinal injection was selected approximately three disc diameters temporal to the macula in a location free of subretinal fibrosis or other pathology. A 41-gauge needle was positioned just above the retina, and $300 \mu \mathrm{L}$ of vector was injected briskly, creating a stream that penetrated the retina, forming a bleb containing the vector. Only on one occasion did the vector stream not penetrate the retina immediately, allowing some vector escape into the vitreous cavity prior to penetration of the retina. To ensure that a total volume of $300 \mu \mathrm{L}$ of vector was injected in the subretinal space in subsequent procedures, the protocol was amended, and balanced salt solution was first injected through the 41-gauge needle to raise a small bleb. The 41-gauge needle was withdrawn, another 41-gauge needle attached to extension tubing and syringe containing vector was inserted into the bleb, and $300 \mu \mathrm{L}$ of vector was injected into the subretinal space. After subretinal injection of vector, a partial fluid-air exchange was done. There was a minimum interval of 14 days between dosing the first and subsequent patients in each cohort and at least 28 days after completing a cohort, at which point the Data and Safety Monitoring committee reviewed all data and determined if it was safe to proceed to the next cohort.

\section{Study visits and procedures}

At each study visit, patients received a complete eye examination, including BCVA and OCT. Fluorescein angiograms were performed at baseline 
and at 12,24 , and 48 weeks. An anterior chamber tap was performed at baseline and at 1, 4, 8, 12, 24, and 48 weeks, and aqueous humor was assayed for endostatin and angiostatin. At each visit, blood and urine samples were obtained for safety laboratory analysis. Samples were also obtained for immune response to vector and PCR assessments for viral particles at selected visits during the study.

Following completion of the 48-week follow up, patients were asked to enter a long-term, openlabel, follow-up protocol in which visits for ophthalmologic examinations and recording of AEs were continued at six-monthly intervals for 5 years and at a minimum interval of once a year for a subsequent 10 years to monitor for delayed AEs. Additionally, as part of this long-term follow-up protocol, blood samples are taken and analyzed using PCR every 6 months for 5 years and then every year for 10 years.

\section{Optical coherence tomography}

Heidelberg Spectralis (Heidelberg Engineering, Inc., Heidelberg, Germany) SD-OCT scans were obtained at each visit. The following scan acquisition parameters were used for Heidelberg Spectralis: volume scan $\left(20^{\circ} \times 20^{\circ}\right.$, roughly $6 \mathrm{~mm} \times$ $6 \mathrm{~mm}$ ) with $25 \mathrm{~B}$-scans in horizontal orientation spaced $240 \mu \mathrm{m}$ apart, minimum automatic realtime mean of 9 , high speed (512 A-scans/B-scan). All scans following the visit on day 1 were acquired with the TruTrack ${ }^{\mathrm{TM}}$ eye tracker using the progression scan function. SD-OCT images were graded for CST with manual correction of algorithms and grid alignment if required, using the Heidelberg Eye Explorer (v1.8.6.0), HRA/Spectralis Viewing Module v5.3.2.0 at the Johns Hopkins University Retinal Imaging Research and Reading Center (Baltimore, MD). Manual measurements were made for the maximum width and height of the subretinal tissue on each section of the scan. The central lesion thickness was determined by measuring the maximum height of the subretinal tissue on the horizontal OCT section through the fovea.

On visits where the Heidelberg Spectralis machine was not available, OCT scans were performed on the Stratus OCT3 machine (Carl Zeiss Meditec, Dublin, CA) using the Fast Macular Scan protocol (6 line scans that are $6 \mathrm{~mm}$ long centered on fixation and spaced $30^{\circ}$ apart around the circumference of a circle; each line consists of 128 A-scan measurements).

\section{Fundus photography and FA}

The fundus photographs (FP) and FA were performed for the required study visits using the modified three-field protocol. The FP images were graded for the presence of subretinal/intraretinal hemorrhage, exudates, and subretinal fibrosis. The FA images were graded for the presence of leakage, and lesion measurements were made using Image J v1.45s (National Institutes of Health, Bethesda, MD; available at http://rsbweb.nih.gov).

\section{Expression studies in rabbits}

Dutch-belted rabbits were treated in in accordance with U.S. Food and Drug Administration Good Laboratory Practice regulations. Subretinal injections were done as previously described ${ }^{32}$ us- $^{-}$ ing an early version of RetinoStat in which the RPE-specific vitelliform macular dystrophy 2 promoter was used to express human forms of endostatin and angiostatin. Injections were done in one eye, and the other eye was left untreated. Briefly, rabbits were anesthetized, pupils were dilated, and under an operating microscope, a 37gauge needle was inserted $2 \mathrm{~mm}$ posterior to the limbus into the vitreous cavity. The needle was positioned just above the retina, and $100 \mu \mathrm{L}$ containing $6.5 \times 10^{5} \mathrm{TU}$ or $6.5 \times 10^{6} \mathrm{TU}$ of vector $(n=4$ for each) was briskly injected penetrating the retina and causing a bleb. Rabbits were euthanized between 3 and 6 weeks, and aqueous humor and vitreous samples were obtained. The concentration of human endostatin in each sample was measured by ELISA.

\section{RESULTS}

\section{Patient demographics, baseline characteristics, and disposition}

Twenty-one subjects with advanced NVAMD $\left(M_{\text {age }}=79.4\right.$ years $)$ were enrolled in three doseranging cohorts $\left(2.4 \times 10^{4} \mathrm{TU}, 2.4 \times 10^{5} \mathrm{TU}\right.$, and $8.0 \times 10^{5} \mathrm{TU} ; n=3$ for each) and a MTD cohort $\left(8.0 \times 10^{5} \mathrm{TU} ; n=12\right)$. As all three doses were welltolerated in the first part, the MTD was the highest dose. Since this was the first experience with RetinoStat in humans, eligibility for the first three cohorts required limited visual potential due to subretinal fibrosis, and mean baseline BCVA for cohorts 1-3 was 21.4 Early Treatment Diabetic Retinopathy Study (ETDRS) letter score (20/400 Snellen equivalent; Table 1). This requirement was relaxed for the MTD cohort for which mean baseline BCVA was 33.0 in ETDRS letter score (20/200). On average, subjects in the MTD cohort had less severe disease, but there were some patients with extensive subretinal fibrosis and poor vision (Supplementary Table S1 and Supplementary Fig. S2). While most patients had received intraocular in- 
Table 1. Demographics and baseline characteristics of study subjects

\begin{tabular}{lcc}
\hline & $\begin{array}{c}\text { Dose escalation } \\
(\mathrm{n}=9)\end{array}$ & $\begin{array}{c}\text { Maximum dose } \\
\text { cohort }(\mathrm{n}=12)\end{array}$ \\
\hline $\begin{array}{l}\text { Mean age (years) } \\
\text { Females, } n \text { (\%) }\end{array}$ & 80.0 & 79.0 \\
Race, $n(\%)$ & $6(66.7)$ & $5(41.7)$ \\
$\quad$ Caucasian & $8(88.9)$ & $9(75.0)$ \\
$\quad$ African American & $0(0.0)$ & $2(16.7)$ \\
$\quad$ Other & $1(11.1)$ & $1(8.3)$ \\
$\begin{array}{c}\text { Duration of AMD } \\
\text { (months), } M \text { (range) }\end{array}$ & $47.9(20-124)$ & $68.3(6-160)$ \\
$\begin{array}{c}\text { Prior anti-VEGF, } \\
\text { patients, } n \text { (\%) }\end{array}$ & $8(88.9)$ & $11(91.7)$ \\
BCVA, letter score \\
$\quad$ (Snellen equivalent)
\end{tabular}

$A M D$, age-related macular degeneration; BCVA, best-corrected visual acuity CST, central subfield thickness; VEGF, vascular endothelial growth factor.

jections of a VEGF-neutralizing protein, subjects in the dose-ranging cohorts were no longer receiving them because of poor visual potential and perceived lack of visual benefit. Most subjects had intraretinal and/or subretinal fluid and ongoing leakage from choroidal NV confirmed by fluorescein angiography. Mean CST (an assessment of intraretinal fluid) measured by SD-OCT was $439.0 \mu \mathrm{m}$ for cohorts $1-3$ and $255.6 \mu \mathrm{m}$ for cohort 4 . All subjects completed the 48-week core study, and

Table 2. Disposition table

\begin{tabular}{|c|c|c|c|c|c|}
\hline Cohort & Dose (TU) & $\begin{array}{l}\text { Study } \\
\text { eye }\end{array}$ & $\begin{array}{l}\text { Core study } \\
\text { completion }\end{array}$ & $\begin{array}{l}\text { Follow-up } \\
\text { duration } \\
\text { (months) }\end{array}$ & $\begin{array}{c}\text { Consented } \\
\text { for long-term } \\
\text { follow-up study }\end{array}$ \\
\hline \multirow[t]{3}{*}{1} & \multirow[t]{3}{*}{$2.4 \times 10^{4}$} & Left & Yes & 12 & Yes \\
\hline & & Right & Yes & 12 & Yes \\
\hline & & Right & Yes & 12 & Yes \\
\hline \multirow[t]{3}{*}{2} & \multirow[t]{3}{*}{$2.4 \times 10^{5}$} & Right & Yes & 12 & Yes \\
\hline & & Left & Yes & 12 & Yes \\
\hline & & Right & Yes & 12 & Yes \\
\hline \multirow[t]{3}{*}{3} & \multirow[t]{3}{*}{$8.0 \times 10^{5}$} & Right & Yes & 12 & No \\
\hline & & Right & Yes & 12 & No \\
\hline & & Left & Yes & 12 & Yes \\
\hline \multirow[t]{12}{*}{4 or MTD } & \multirow[t]{12}{*}{$8.0 \times 10^{5}$} & Left & Yes & 12 & Yes \\
\hline & & Right & Yes & 12 & Yes \\
\hline & & Right & Yes & 12 & Yes \\
\hline & & Right & Yes & 12 & Yes \\
\hline & & Left & Yes & 12 & Yes \\
\hline & & Left & Yes & 12 & Yes \\
\hline & & Right & Yes & 12 & Yes \\
\hline & & Left & Yes & 12 & Yes \\
\hline & & Left & Yes & 12 & Yes \\
\hline & & Left & Yes & 12 & No \\
\hline & & Right & Yes & 12 & Yes \\
\hline & & Right & Yes & 12 & Yes \\
\hline
\end{tabular}

MTD, maximum tolerated dose; TU, transducing units.
Table 3. Ocular adverse events

\begin{tabular}{llc}
\hline Ocular adverse events & $\begin{array}{c}\text { Cohort 1+2, } \\
\mathrm{N}=6(\%)\end{array}$ & $\begin{array}{c}\text { Cohort 3+4, } \\
\mathrm{N}=15(\%)\end{array}$ \\
\hline Anterior chamber cell & $0(0.0)$ & $1(6.7)$ \\
Cataract development/progression & $2(33.3)$ & $6(40.0)$ \\
Choroidal neovascularization & $0(0.0)$ & $2(13.3)$ \\
Corneal edema & $0(0.0)$ & $1(6.7)$ \\
Eye pain & $0(0.0)$ & $4(26.7)$ \\
Eye pruritus & $1(16.7)$ & $2(13.3)$ \\
IOP elevation & $0(0.0)$ & $1(6.7)$ \\
Intra/subretinal hemorrhage & $3(50)$ & $8(53.3)$ \\
Macular hole & $0(0.0)$ & $1(6.7)$ \\
Macular edema & $0(0.0)$ & $3(20.0)$ \\
Retinal tear & $0(0.0)$ & $2(13.3)$ \\
Subconjunctival hemorrhage & $0(0.0)$ & $3(20.0)$ \\
Subretinal fibrosis & $0(0.0)$ & $1(6.7)$ \\
Subretinal fluid & $0(0.0)$ & $2(13.3)$ \\
Vision blurred & $1(16.7)$ & $1(6.7)$ \\
Visual acuity reduced & $0(0.0)$ & $3(20.0)$ \\
Vitreous floaters & $0(0.0)$ & $2(13.3)$ \\
Vitreous hemorrhage & $0(0.0)$ & $1(6.7)$ \\
\hline
\end{tabular}

IOP, intraocular pressure.

18 subjects entered the long-term follow-up study (Table 2). Reported herein are the safety, anatomical, and visual outcomes of all patients during the 48-week core study. Transgene expression data are reported for all subjects through 48 weeks and for some subjects through their most recent visit in the long-term study (1.5-4.5 years).

\section{Safety}

There was no dose-limiting toxicity during the Dose Escalation Phase, and therefore the highest dose, $8.0 \times 10^{5} \mathrm{TU}$, was utilized in the MTD cohort. A total of 15 subjects, three in cohort 3 and 12 in the MTD cohort, received a subretinal injection of $8.0 \times 10^{5}$ TU. One intraoperative serious and two non-serious AEs occurred, and were clearly complications of the study procedure for vector administration (Tables 3 and 4). In one subject, a macular hole occurred during subretinal injection of vector. A fluid-air exchange was done at the end of the procedure, and the macular hole was sealed after the air resorbed. In two other subjects, a peripheral retinal tear was identified at the end of the

Table 4. Serious adverse events

\begin{tabular}{lcc}
\hline Serious adverse events & $\begin{array}{c}\text { Cohort 1+2, } \\
\mathrm{N}=6(\%)\end{array}$ & $\begin{array}{c}\text { Cohort 3+4, } \\
\mathrm{N}=15(\%)\end{array}$ \\
\hline Intraductal proliferative breast lesion & $1(16.7)$ & $0(0)$ \\
Macular hole & $0(0)$ & $1(6.7)$ \\
Osteoarthritis & $1(16.7)$ & $0(0)$ \\
Pneumonia & $1(16.7)$ & $0(0)$ \\
Pneumothorax & $0(0)$ & $1(6.7)$ \\
Urinary tract infection & $0(0)$ & $1(6.7)$ \\
\hline
\end{tabular}


procedure, and it was felt that each of the tears occurred during dissection and removal of the posterior hyaloid. The tears were treated with retinopexy and fluid-air exchange, resulting in sealing of the tears and no related problems during the remainder of the trial. Several other AEs were noted in the early postoperative period and were felt likely related to study procedure for vector administration, including mild corneal edema, eye pain, pruritus, elevation of IOP, and mild vitreous hemorrhage (Table 3). Although a greater range occurred in the high-dose group, there was not a large difference in the number of patients experiencing each event, and all were deemed unrelated to the RetinoStat vector. So this finding is due to the greater patient numbers receiving the high dose rather than the higher dose itself. All of these resolved rapidly without sequelae. Six subjects experienced worsening of cataract, a known late complication of vitreous surgery. Several AEs were felt to be related to progression of NVAMD, including subretinal hemorrhage, fibrosis, and/or fluid, and reduced vision. The subject who experienced the macular hole was noted to have anterior chamber cells in the early postoperative period that cleared rapidly and was deemed to have been related to the procedure. However, the mild and transient nature of the inflammation in this subject and lack of any inflammation in all other subjects suggest that subretinal injection of EIAV vector is well-tolerated with minimal if any immune response. Also notable is that the subretinal injection procedure was well-tolerated with no complications other than the macular hole and retinal tears, which were addressed and resolved.

There were no deaths during the 48-week core study. There were six serious AEs that were judged unlikely related to RetinoStat, but one, the macular hole, was related to study procedure (Table 4). Non-ocular AEs were felt unrelated to RetinoStat or study procedure (Supplementary Table S2).

\section{Immune response and biodistribution}

Antibodies to RetinoStat were not detected in any patients. Using a sensitive PCR assay, RetinoStat RNA sequences were detected in the plasma of one subject on day 0 but not thereafter, and no sequences were ever detected in buffy coat or urine at any time points.

\section{Transgene expression}

There were no detectable levels of endostatin in the aqueous humor of subjects at baseline. Subjects in cohort 1 had detectable low levels at week 4 through week 48 (Fig. 1). Subjects in cohorts 2-4 had measurable levels of endostatin at week 1 , which increased substantially at week 4 and peaked at mean levels of $57-81 \mathrm{ng} / \mathrm{mL}$ between 12 and 24 weeks. The change in mean levels of angiostatin in aqueous humor mirrored those seen for endostatin (although consistently lower, due in part to the position of the angiostatin gene after the IRES element in the expression cassette), with a small increase for cohort 1 and substantial increases for cohorts $2-4$, which peaked at $15-27 \mathrm{ng} /$ $\mathrm{mL}$ by weeks $12-24$. As shown by the small error bars, the variability in transgene expression among patients within each dose level at each time point was small.

Aqueous humor levels of proteins secreted from RPE cells and photoreceptors are substantially less than levels in the retina, while vitreous levels provide a closer approximation. In rabbits, after subretinal injection of $6.5 \times 10^{5} \mathrm{TU}$ or $6.5 \times 10^{6} \mathrm{TU}$ of an early version of RetinoStat, which utilized the retinal pigmented epithelium-specific vitelliform macular dystrophy 2 promoter, vitreous levels of endostatin were 34- and 30-fold higher than those in aqueous humor, respectively (Supplementary Fig. S3). This suggests that steady state aqueous humor levels of $15-27 \mathrm{ng} / \mathrm{mL}$ in eyes of patients in cohorts 2-4 likely correspond to vitreous levels of approximately $450-810 \mathrm{ng} / \mathrm{mL}$.

In order to assess long-term expression beyond week 48, available samples from subjects with significant levels of expression (higher than seen in the low-dose group, cohort 1) were measured during the long-term follow-up study. Samples were not obtained from three subjects (3A, $3 \mathrm{~B}$, and $4 \mathrm{~J}$ ) because one declined entry into the long-term follow-up study, one refused aqueous humor taps, and one died during long-term follow-up from causes judged unrelated to study drug or procedure. To date, endostatin and angiostatin have been measured in 11 subjects (all three subjects in cohort 2 and eight in cohort 4; Fig. 2). Expression is detectable at the last measurement in cohort 2 subjects $(2.5,4$, and 4.5 years $)$ and cohort 4 subjects (1.5-2.5 years). Compared to levels at month 12 , expression has remained relatively stable (or increased) for both transgenes in all subjects. Therefore, expression observed in the main study has persisted during long-term follow-up, including one subject with particularly long follow-up in which a sample tested was obtained 4.5 years after vector injection.

\section{Anatomic outcomes}

For subjects who did not receive any anti-VEGF injections, one in each of the cohorts showed a 

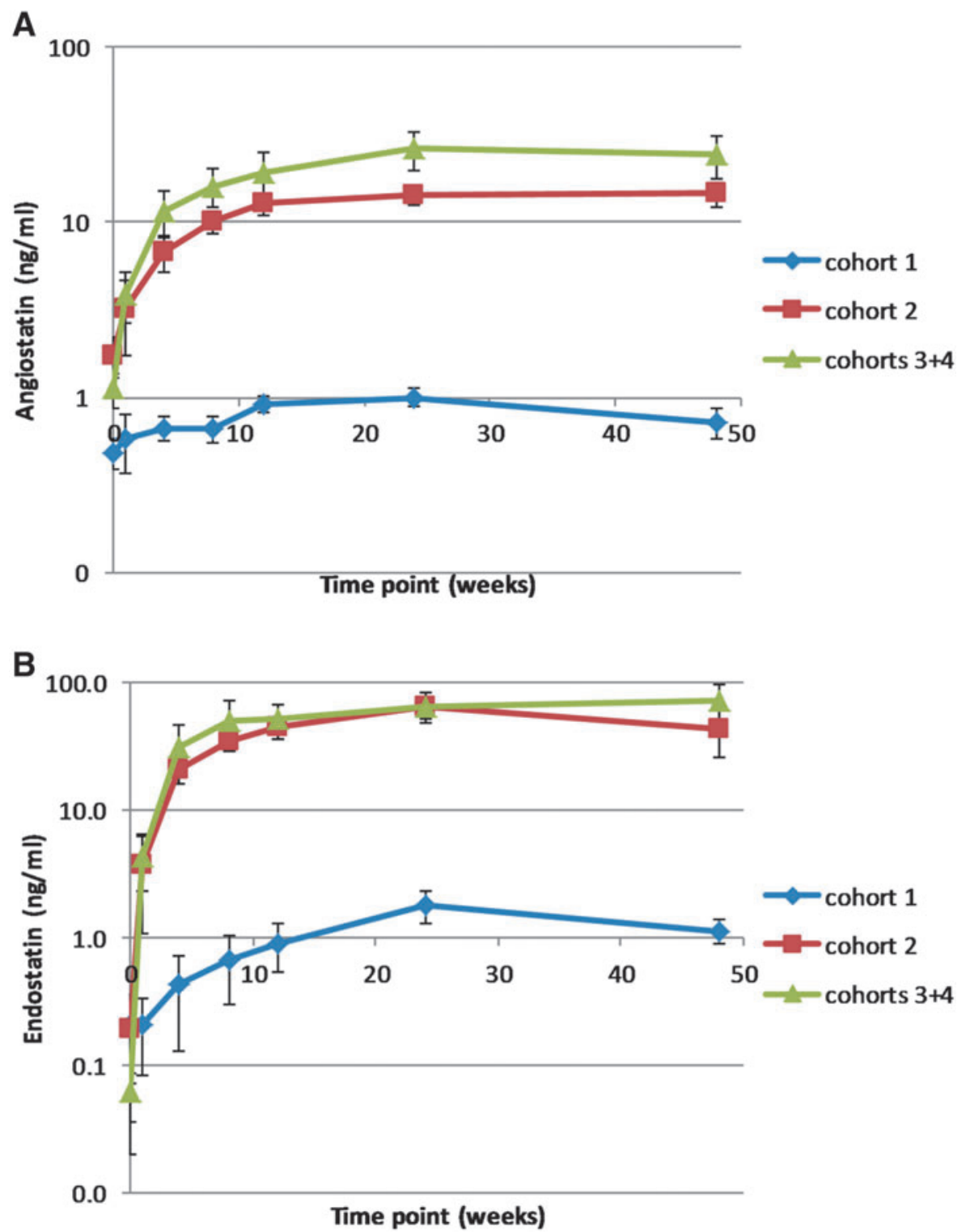

Figure 1. Aqueous levels of angiostatin (A) and endostatin (B) after subretinal injection of RetinoStat ${ }^{\circledR}$. Aqueous samples were obtained at baseline and at 1 , $4,8,12,24$, and 48 weeks after injection of $2.4 \times 10^{4}$ transduction units (TU; cohort $1 ; n=3$ ), $2.4 \times 10^{5} \mathrm{TU}$ (cohort $2 ; n=3$ ), or $8.0 \times 10^{5} \mathrm{TU}$ (cohorts 3 and $4 ; n=15$ ). Endostatin and angiostatin were measured by semi-quantitative Western blot analysis and relative densitometry. Mean ( \pm standard error of the mean) expression was low in cohort 1, but subjects in cohorts 2-4 had measurable levels of endostatin at week 1, which increased substantially at week 4, peaked at $57-81 \mathrm{ng} / \mathrm{mL}$ between weeks 12 and 24 , and were maintained through week 48. Levels of angiostatin for cohorts $2-4$ peaked at $15-27 \mathrm{ng} / \mathrm{mL}$ between weeks 24 and 48 and remained stable through week 48.

substantial reduction from baseline CST at week 48 , but the others showed no change or increases (Fig. 3A-D). Seven subjects in cohort 4 received anti-VEGF injections due to persistent or increased disease activity (Table 5). Subject $4 \mathrm{~K}$ received antiVEGF injections at weeks $8,16,20$, and 42 , and showed a substantial reduction in CST at week 48 , but the others showed little change in CST (Fig. 3E). In the 15 subjects from cohorts 3 and 4 who received the highest vector dose, there was no mean change from baseline CST at week 48 (Fig. 3F).
The change from baseline CST does not provide a complete picture because it is valuable to know the amount of fluid in or under the macula at baseline and how it changes over time. Therefore, sequential SD-OCTs for all subjects are shown in Supplementary Fig. S4. Subject 1B in cohort 1 who showed a CST reduction from 716 to $558 \mu \mathrm{m}$ and thus had severe residual thickening. Subject $2 \mathrm{~B}$ in cohort 2 showed gradual reduction of fluid with complete elimination at week 16 and beyond. In the 15 subjects injected with $8.0 \times 10^{5} \mathrm{TU}$, three were uninformative because they lacked fluid at base- 


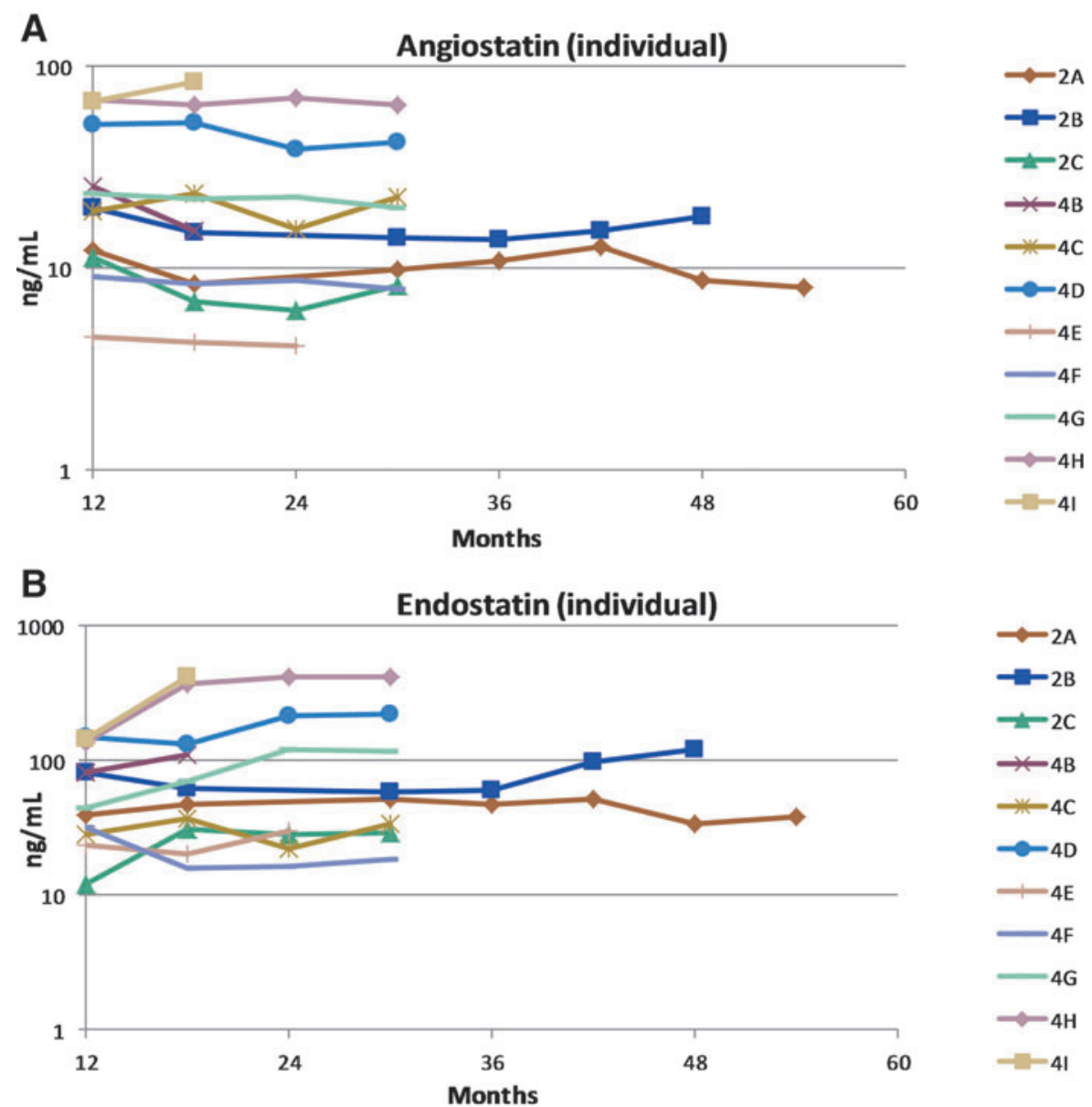

Figure 2. Aqueous levels of endostatin and angiostatin after subretinal injection of RetinoStat in the long-term follow-up study. Aqueous samples were obtained every 6 months (scheduled up to 5 years, then annually thereafter). Endostatin (A) and angiostatin (B) were measured by semi-quantitative Western blot analysis and relative densitometry for patients with significant levels of expression during the main study. Expression remained stable or increased over time.

line and never developed it, three lacked fluid at baseline and subsequently developed it, seven had intraretinal or subretinal fluid at baseline that was not reduced, and two had reductions in fluid attributable to anti-VEGF injections. Therefore, only one subject (2B) showed clear evidence of antipermeability activity.

The FP of subjects in cohorts 1-3 at baseline (Supplementary Fig. S2) illustrate considerable subretinal fibrosis, with one subject showing subretinal hemorrhage (1A) and three showing subretinal exudates $(1 \mathrm{~B}, 3 \mathrm{~B}$, and $3 \mathrm{C})$, a sign of severe and prolonged leakage. At week 48, subretinal fibrosis and overall lesion size did not appear to be substantially enlarged, but the three subjects with subretinal exudates at baseline showed an increase in exudates indicating persistent leakage. Subjects in cohort 4 also had limited visual prognosis due to subretinal fibrosis, but some subjects had relatively small areas of fibrosis. While most subjects showed little change in overall lesion size at week 48 , one subject showed a definite increase with surrounding subretinal hemorrhage indicating persistent disease activity (Supplementary Fig. S2 and patient $4 \mathrm{~K})$. Two other subjects also showed subretinal hemorrhage at week 48 (1B and $4 \mathrm{~L}$ ). Mean lesion sizes at baseline and at week 48 were $6.7 \pm 2.7$ and $7.1 \pm 1.1$ disc areas for cohort 1, 9.6 \pm 3.0 and $6.5 \pm 4.4$ disc areas for cohort 2 , and $6.3 \pm 1.3$ and $7.4 \pm 1.3$ disc areas for cohorts 3 and 4 .

Leakage during fluorescein angiography, defined as an increase in area of hyperfluorescence between the transit phase and late recirculation phase, was present in all 21 patients at baseline, and at week 24 was present in six (29\%) patients. Reduction in leakage broadly correlated with aqueous humor endostatin/angiostatin levels (Supplementary Fig. S5). However, this did not lead to a subsequent anatomical benefit in the majority of these patients with advanced NVAMD. 

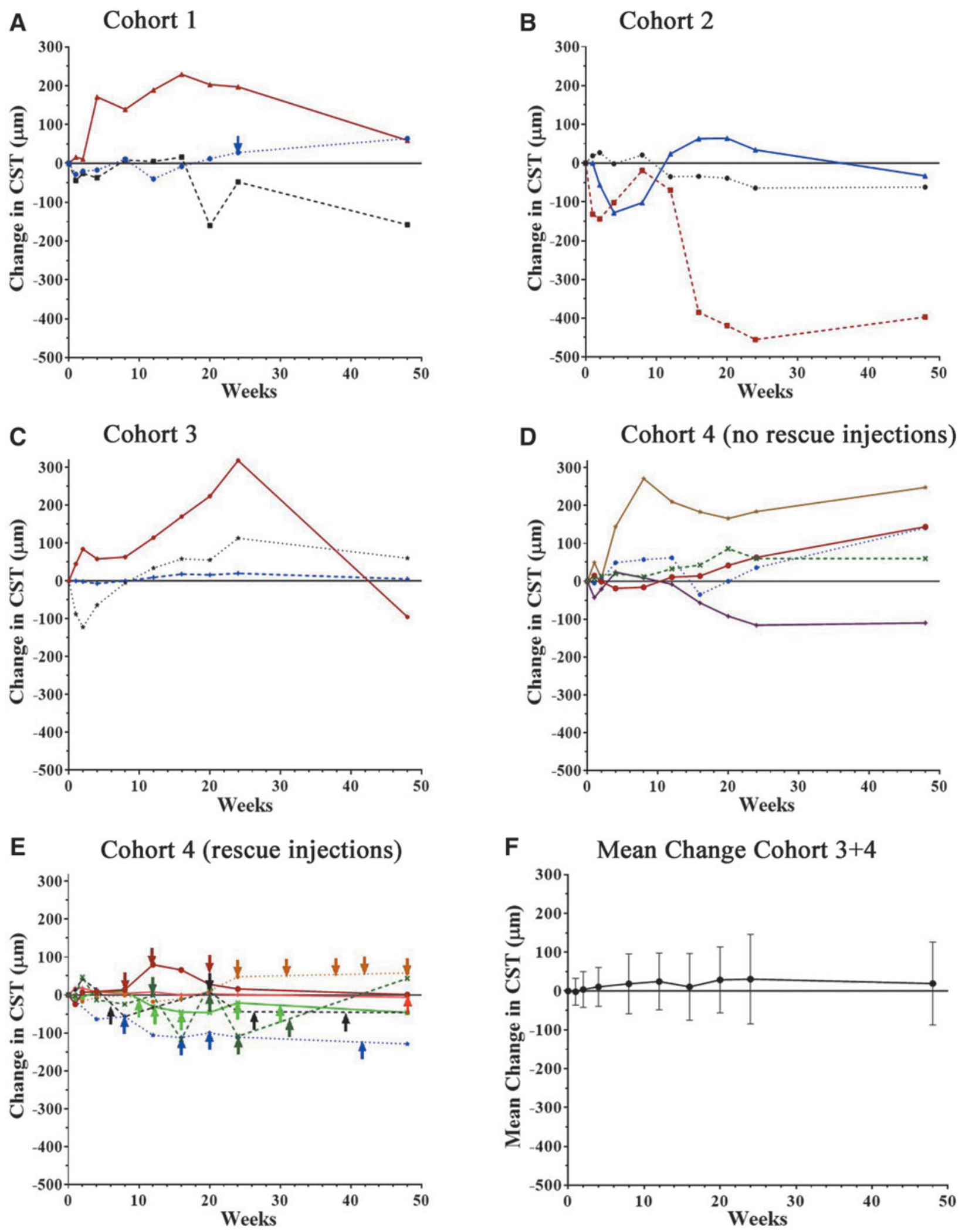

Figure 3. Change from baseline central subfield thickness (CST) after subretinal injection of RetinoStat. Subjects had measurement of CST by optical coherence tomography at baseline and at 1,2,4,8,12, 16, 20,24, and 48 weeks after subretinal injection of $2.4 \times 10^{4} \mathrm{TU}\left(\mathbf{A}\right.$, cohort 1), $2.4 \times 10^{5} \mathrm{TU}(\mathbf{B}$, cohort 2), or $8.0 \times 10^{5} \mathrm{TU}(\mathbf{C}$, cohort 3; $\mathbf{D}$ and $\mathbf{E}$, cohort 4) of RetinoStat. Subjects in cohort 4 are divided into those that did not receive any anti-VEGF injections (D) and those that received at least one anti-VEGF injection (E). There was little mean change ( \pm standard deviation [SD]) in CST between baseline and week 48 in the 15 patients in cohorts 3 and 4 who had a subretinal injection of $8.0 \times 10^{5} \mathrm{TU}$ of RetinoStat $(\mathbf{F})$. 
Table 5. Anti-VEGF injections given in study eye

\begin{tabular}{|c|c|c|c|c|}
\hline Cohort & $\begin{array}{c}\text { Patient } \\
I D\end{array}$ & $\begin{array}{c}\text { Time } \\
\text { since } \\
\text { enrollment } \\
\text { (weeks) }\end{array}$ & $\begin{array}{c}\text { Preferred } \\
\text { anti-VEGF } \\
\text { therapy }\end{array}$ & Indication \\
\hline 1 & $1 \mathrm{~A}$ & 24 & Ranibizumab & Intraretinal or subretinal fluid \\
\hline \multirow[t]{26}{*}{4} & $4 \mathrm{E}$ & 24 & Aflibercept & Intraretinal or subretinal fluid \\
\hline & & 31 & Aflibercept & Intraretinal or subretinal fluid \\
\hline & & 38 & Aflibercept & Intraretinal or subretinal fluid \\
\hline & & 42 & Aflibercept & Intraretinal or subretinal fluid \\
\hline & & 48 & Aflibercept & Intraretinal or subretinal fluid \\
\hline & $4 \mathrm{G}$ & 12 & Aflibercept & Intraretinal or subretinal fluid \\
\hline & & 20 & Aflibercept & Intraretinal or subretinal fluid \\
\hline & & 24 & Aflibercept & Intraretinal or subretinal fluid \\
\hline & & 32 & Aflibercept & Intraretinal or subretinal fluid \\
\hline & $4 \mathrm{H}$ & 8 & Aflibercept & Intraretinal or subretinal fluid \\
\hline & & 12 & Aflibercept & Intraretinal or subretinal fluid \\
\hline & & 20 & Aflibercept & Intraretinal or subretinal fluid \\
\hline & $4 \mid$ & 48 & Aflibercept & Subretinal hemorrhage \\
\hline & $4 \mathrm{~J}$ & 10 & Bevacizumab & Subretinal hemorrhage \\
\hline & & 12 & Bevacizumab & Subretinal hemorrhage \\
\hline & & 16 & Bevacizumab & Subretinal hemorrhage \\
\hline & & 24 & Bevacizumab & Subretinal hemorrhage \\
\hline & & 30 & Bevacizumab & Subretinal hemorrhage \\
\hline & $4 \mathrm{~K}$ & 8 & Bevacizumab & Intraretinal or subretinal fluid \\
\hline & & 16 & Bevacizumab & Intraretinal or subretinal fluid \\
\hline & & 20 & Aflibercept & Intraretinal or subretinal fluid \\
\hline & & 42 & Aflibercept & Subretinal hemorrhage \\
\hline & $4 \mathrm{~L}$ & 6 & Aflibercept & Intraretinal or subretinal fluid \\
\hline & & 20 & Aflibercept & Intraretinal or subretinal fluid \\
\hline & & 26 & Aflibercept & Intraretinal or subretinal fluid \\
\hline & & 39 & Aflibercept & Intraretinal or subretinal fluid \\
\hline
\end{tabular}

\section{Visual outcomes}

Subjects had limited visual potential, and therefore little or no improvement was expected. Two of three subjects in the first cohort showed reduction in BCVA at week 48 (Fig. 4A), and subjects in cohort 2 showed little change (Fig. 4B). Three subjects in cohorts 3 and 4 who did not receive any anti-VEGF injections showed improvement of 10 or more letters in BCVA, but one showed a substantial reduction (Fig. 4C and D). Cohort 4 subjects who received anti-VEGF injections tended to show reductions in BCVA despite the injections (Fig. 4E), and for all 15 subjects in cohorts 3 and 4 who received the highest vector dose, there was no mean change from baseline BCVA at week 48 (Fig. 4F).

\section{DISCUSSION}

The eye is a relatively isolated organ, which makes it ideally suited for gene transfer. Previous studies have demonstrated that intravitreal injection of an adenoviral vector ${ }^{33}$ or subretinal injection of an adeno-associated viral vector ${ }^{34-37}$ is well-tolerated. This study provides the first demonstration that subretinal injection of an EIAV lentiviral vector is also safe and well-tolerated. While transgene expression has been implied in previous gene therapy studies by evidence of biologic effects, the level, reproducibility, and duration of expression is unknown, and in some studies the biological effect has not been sustained. ${ }^{38,39}$ This is the first study to provide direct measurement of transgene expression, and demonstrates that subretinal injection of an EIAV vector containing an expression construct in which a CMV promoter drives expression of two secreted proteins results in sustained high levels of the two proteins in the eye. These data are consistent with expression studies in rabbits. ${ }^{32}$ The reproducibility of significant transgene levels among patients in each cohort at each time point suggests that subretinal vector injections are quite reliable. Two different approaches for subretinal injection were employed. Initially, the vector was used to penetrate the retina, but in one subject, there was a delay in penetration of the retina so that some vector escaped into the vitreous cavity, and transgene expression was lower in that subject than others in the cohort. The protocol was modified so that a small bleb was created with balanced salt solution, and then the vector was injected into the bleb to ensure that the entire $300 \mu \mathrm{L}$ of vector was injected into the subretinal space. Expression was good in most cases with either approach. However, 4/15 subjects who received the highest dose had endostatin and angiostatin levels close to those seen in the low-dose cohort. Of these, two experienced intraoperative complications (macular hole and retinal tear). However, in general, there was a good correlation between vector dose and mean protein concentrations. The peak mean levels of $>80 \mathrm{ng} / \mathrm{mL}$ of endostatin at the highest dose and the maintenance of these levels in the majority of patients for at least 48 weeks is impressive, particularly as data generated in the rabbit indicate levels in the retina are likely to be at least 30 -fold higher than those in aqueous humor (Supplementary Fig. S3). Moreover, the continued detection of transgene products in samples from the ongoing long-term follow-up study for up to 4.5 years strongly supports the use of this platform for the treatment of chronic disease.

Assessment of biologic effect is challenging in a safety study involving patients with advanced NVAMD who have irreversible subretinal fibrosis, making it impossible to achieve lesion resolution. However, despite a large component of irreversible disease, intraretinal and subretinal fluid implies active choroidal NV that allows testing of antipermeability and anti-angiogenic activity. Despite 


\section{A Cohort 1}

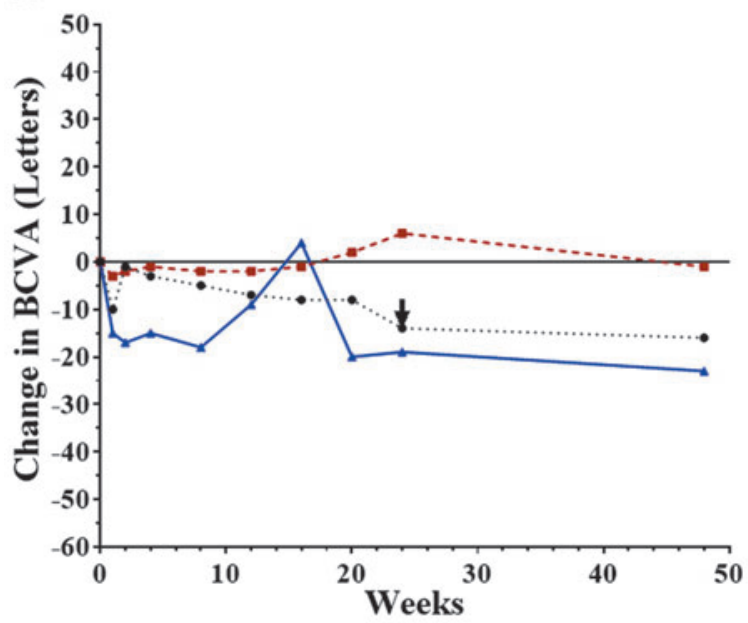

C Cohort 3
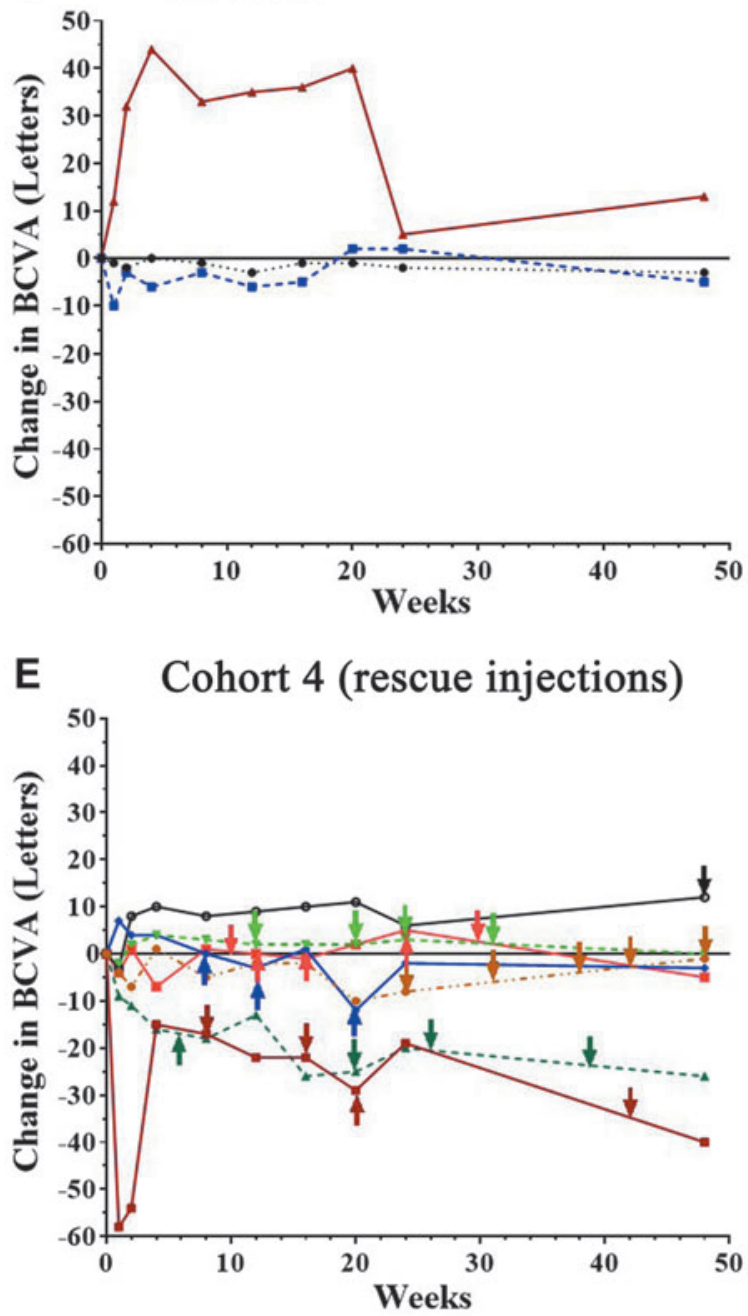

B

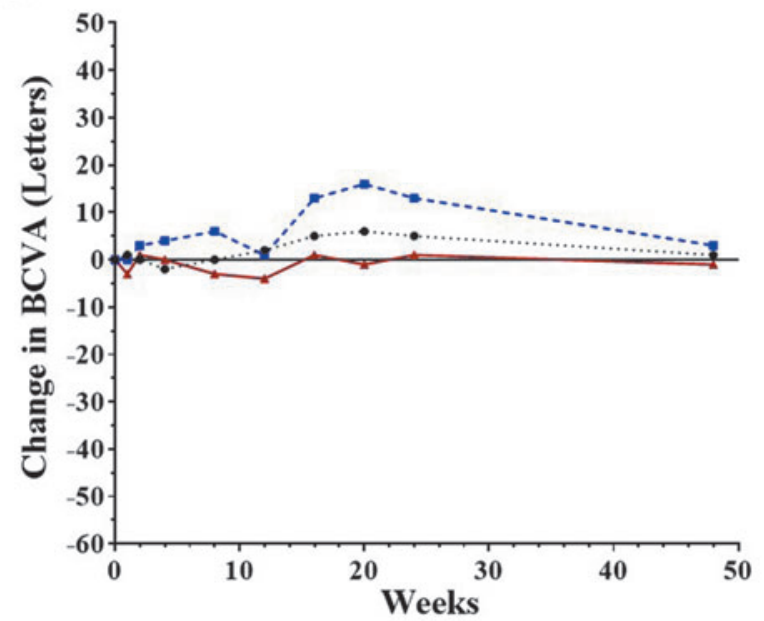

D Cohort 4 (no rescue injections)

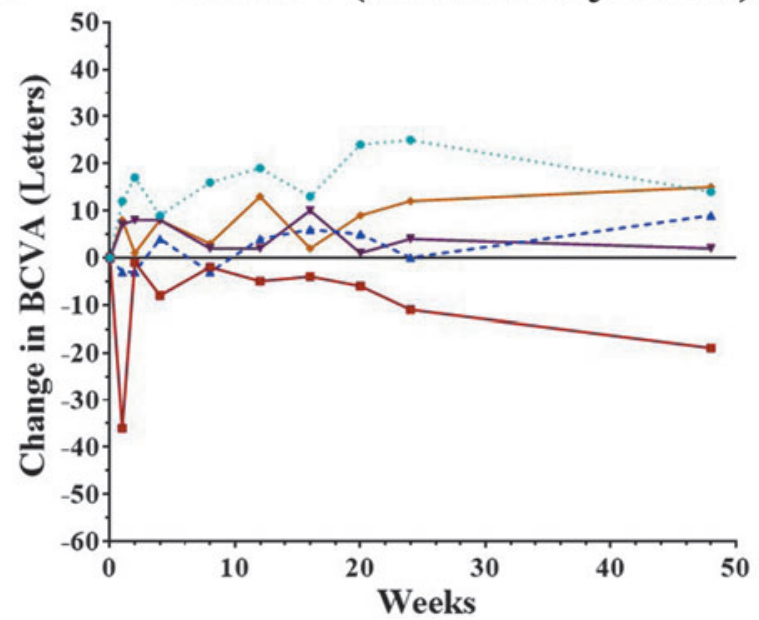

F Mean Change Cohort 3+4

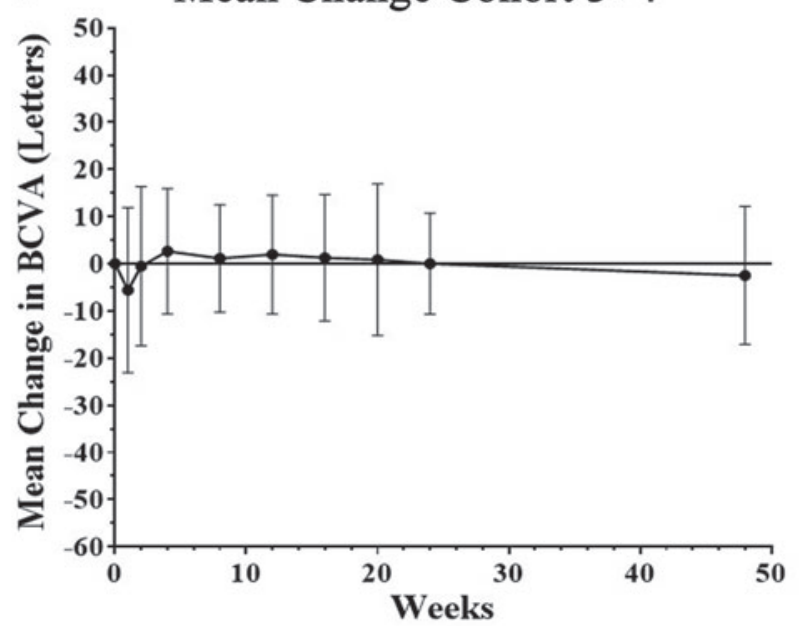

Figure 4. Change from baseline best corrected visual acuity (BCVA) after subretinal injection of RetinoStat. Subjects had measurement of $B C V A$ at baseline and at $1,2,4,8,12,16,20,24$, and 48 weeks after subretinal injection of $2.4 \times 10^{4} \mathrm{TU}\left(\mathbf{A}\right.$, cohort 1), $2.4 \times 10^{5} \mathrm{TU}\left(\mathbf{B}\right.$, cohort 2), or $8.0 \times 10^{5} \mathrm{TU}(\mathbf{C}$, cohort $3 ; \mathbf{D}$ and $\mathbf{E}$, cohort 4) of RetinoStat. Subjects in cohort 4 are divided into those that did not receive any anti-VEGF injections (D) and those that received at least one antiVEGF injection $(E)$. There was little mean change $( \pm S D)$ in BCVA between baseline and week 48 in the 15 patients in cohorts 3 and 4 who had a subretinal injection of $8.0 \times 10^{5} \mathrm{TU}$ of RetinoStat (F). 
reduction in fluorescein angiographic leakage, the data failed to provide strong evidence that endostatin or angiostatin has a therapeutic benefit in the majority of these advanced NVAMD subjects. An endostatin- and/or angiostatin-induced antipermeability effect cannot be ruled out in one patient, but there were many subjects who could have demonstrated an anti-permeability effect if one existed and did not. It is possible that these patients with advanced disease are not predictive of outcomes in patients with early-stage NVAMD, which can only be answered by an additional clinical trial.

In summary, the present data demonstrate that subretinal injection of EIAV is safe and welltolerated, and provides reproducible, sustained transgene expression. EIAV-based lentiviral vectors are an outstanding platform for gene replacement or gene delivery in the eye. This study has also demonstrated that sustained expression of high levels of endostatin and angiostatin in the eye may reduce fluorescein angiographic leakage, but does not reliably eliminate subretinal and/or intraretinal fluid in patients with advanced NVAMD. The data provide a strong rationale for utilization of EIAV-based vectors to treat chronic ocular diseases.

\section{ACKNOWLEDGMENTS} Ltd.

The study was funded by Oxford BioMedica (UK)

\section{AUTHOR DISCLOSURE}

S.E., R.H., M.K., S.N., M.C.A., and K.A.M. are employees or former employees of Oxford BioMedica, which funded this study. They and their families have ownership interests in the company. A.L. was a consultant for Oxford BioMedica. The remaining authors, including those responsible for the assessment of study eligibility and for the clinical measurements and statistical analyses, have no involvement in Oxford BioMedica and declare that they have no conflicts of interest.

\section{REFERENCES}

1. Klein R, Klein BE, Tomany SC, et al. Ten-year incidence and progression of age-related macular degeneration: the Beaver Dam Eye study. Ophthalmology 2002;109:1767-1779.

2. Klein ML, Ferris FLI, Armstrong J, et al. Retina precursors and the development of geographic atrophy in age-related macular degeneration. Ophthalmology 2008;115:1026-1031.

3. Chew EY, Clemons TE, Agron E, et al. Ten-year follow-up of age-related macular degeneration in the age-related eye disease study. JAMA Ophthalmol 2014;132:272-277.

4. Rosenfeld PJ, Brown DM, Heier JS, et al. Ranibizumab for neovascular age-related macular degeneration. N Eng J Med 2006;355:1419-1431.

5. Dawson DW, Volpert OV, Gillis P, et al. Pigment epithelium-derived factor: a potent inhibitor of angiogenesis. Science 1999;285:245-248.

6. O'Reilly MS, Boehm T, Shing Y, et al. Endostatin: an endogenous inhibitor of angiogenesis and tumor growth. Cell 1997;88:277-285.

7. O'Reilly MS, Holmgren S, Shing Y, et al. Angiostatin: a novel angiogenesis inhibitor that mediates the suppression of metastases by a Lewis lung carcinoma. Cell 1994;79:315-328.

8. Kwak N, Okamoto N, Wood JM, et al. VEGF is an important stimulator in a model of choroidal neovascularization. Invest Ophthalmol Vis Sci 2000; 41:3158-3164.
9. Saishin Y, Saishin Y, Takahashi K, et al. VEGFTRAP $_{\text {R1R2 }}$ suppresses choroidal neovascularization and VEGF-induced breakdown of the blood-retinal barrier. J Cell Physiol 2003;195:241-248.

10. Mori K, Duh E, Gehlbach P, et al. Pigment epithelium-derived factor inhibits retinal and choroidal neovascularization. J Cell Physiol 2001; 188:253-263.

11. Mori K, Gehlbach P, Yamamoto $S$, et al. AAV-mediated gene transfer of pigment epitheliumderived factor inhibits choroidal neovascularization. Invest Ophthalmol Vis Sci 2002;43: 1994-2000.

12. Mori $K$, Ando A, Gehlbach $P$, et al. Inhibition of choroidal neovascularization by intravenous injection of adenoviral vectors expressing secretable endostatin. Am J Pathol 2001;159:313-320.

13. Takahashi K, Saishin Y, Saishin $Y$, et al. Intraocular expression of endostatin reduces VEGFinduced retinal vascular permeability, neovascularization, and retinal detachment. FASEB J 2003; 17:896-898.

14. Lai C-C, Wu W-C, Chen S-L, et al. Suppression of choroidal neovascularization by adeno-associated virus vector expressing angiostatin. Invest Ophthalmol Vis Sci 2001;42:2401-2407.

15. Campochiaro PA. Gene transfer for neovascular age-related macular degeneration. Hum Gene Ther 2011;22:523-529
16. Mitrophanous K, Yoon S, Rohll J, et al. Stable gene transfer to the nervous system using a non-primate lentiviral vector. Gene Ther 1999;6:1808-1818.

17. Wong LF, Goodhead L, Prat C, et al. Lentivirusmideiated gene transfer to the central nervous system: therapeutic and research applications. Jun Gene Ther 2006;17:1-9.

18. Kordower JH, Bloch J, Ma SY, et al. Lentiviral gene transfer to the nonhuman primate brain. Exp Neurol 1999;160:1-16.

19. Kordower JH, Emborg ME, Bloch J, et al. Neurodegeneration prevented by lentiviral vector delivery of GDNF in primate models of Parkinson's disease. Science 2000;290:767-773

20. Azzouz M, Marin-Rendon E, Barber RD, et al Multicistornic lentiviral vector-mediated striatal gene transfer of aromatic L-amino acid decarboxylase, tyrosine hydroxylase, and GTP cyclohydrolase I induces sustained transgene expression, dopamine production, and functional improvement in a rat model of Parkinson's disease. J Neurosci 2002; 22:10302-10312.

21. Binley K, Widdowson P, Loader J, et al. Transduction of photoreceptors with Equine Infectious Anemia Virus lentiviral vectors: safety and biodistribution of StarGen for Stargardt disease. Invest Ophthalmol Vis Sci 2015;546:4061-4071.

22. Lotery AJ, Derksen TA, Russell SR, et al. Gene transfer to the nonhuman primate retina with 
recombinant feline immunodeficiency virus vectors. Hum Gene Ther 2002;13:689-696.

23. Zallocchi M, Binley K, Lad Y, et al. ElAV-based retinal gene therapy in the shaker1 mouse model for usher syndrome type 1B: development of UshStat. PLOS ONE 2014;9:e94272.

24. Azzouz M, Kingsman SM, Mazarakis ND. Lentiviral vectors for treating and modeling human CNS disorders. J Gene Med 2004;6:951-962.

25. Mitrophanous K, Yoon S, Rohil J, et al. Stable gene transfer to the nervous system using a nonprimate lentiviral vector. Gene Ther 1999;6:18081818.

26. Mazarakis ND, Azzouz M, Rohll JB, et al. Rabies virus glycoprotein pseudotyping of lentiviral vectors enables retrograde axonal transprot and access to the nervous system after peripheral delivery. Hum Mol Genet 2001;10:2109-2121.

27. Palfi S, Gurruchaga JM, Ralph GS, et al. Longterm safety and tolerability of ProSavin, a lentiviral vector-based gene therapy for Parkinson's disease: a dose escalaton, open-label phase $1 / 2$ trial. Lancet 2014;383:1138-1146.

28. Balaggan KS, Binley K, Esapa M, et al. Stable and efficient introcular gene transfer using pseudotyped EIAV lentiviral vectors. J Gene Med 2006; 8:275-285.
29. Balaggan KS, Binley K, Esapa $M$, et al. EIAV vector-mediated delivery of endostatin or angiostatin inhibits angiogenesis and vascular hyperpermeability in experimental CNV. Gene Ther 2006;13:1153-1165.

30. Kachi S, Binley K, Yokoi K, et al. EIAV vectormediated co-delivery of endostatin and angiostatin driven by the RPE-specific VMD2 promoter inhibits choroidal neovascularization. Hum Gene Ther 2008;20:31-39.

31. Early Treatment Diabetic Retinopathy Study Group. Photocoagulation for diabetic macular edema. Early Treatment Diabetic Retinopathy Study report number 1. Arch Ophthalmol 1985; 103:1796-1806.

32. Binley K, Widdowson PS, Kelleher M, et al. Safety and biodistribution of an Equine Infectious Anemia Virus-based therapy, RetinoStat, for agerelated macular degeneration. Hum Gene Ther 2012; 23:980-991.

33. Campochiaro PA, Nguyen QD, Shah SM, et al. Adenoviral vector-delivered pigment epitheliumderived factor for neovascular age-related macular degeneration: results of a Phase I clinical trial. Hum Gene Ther 2006;17:167-176.

34. Maguire AM, Simonelli F, Pierce EA, et al. Safety and eficacy of gene transfer for Leber's congenital amaurosis. N Eng J Med 2008;358:2240-2248.
35. Bainbridge JW, Smith AJ, Barker SS, et al. Effect of gene therapy on visual function in Leber's congenital amaurosis. N Eng J Med 2008;358: 2231-2239

36. Cideciyan AV, Alelman TS, Boye SL, et al. Human gene therapy for RPE65 isomerase deficiency activates the retinioid cycle of vision but with slow rod kinetics. Proc Natl Acad Sci U S A 2008; 105:15112-15117.

37. MacLaren RE, Groppe M, Barnard AR, et al. Retinal gene therapy in patient with choroideremia: initial findings from a Phase 1/2 clinical trial. Lancet 2014;383:1129-1137.

38. Bainbridge JW, Mehat MS, Sundaram V, et al Long-term effect of gene therapy on Leber's congenital amaurosis. N Engl J Med 2015;372: 1887-1897.

39. Jacobson SG, Cideciyan AV, Roman AJ, et al. Improvement and decline in vision with gene therapy in childhood blindness. N Engl J Med 2015;372:1920-1926.

Received for publication August 5, 2016; accepted after revision September 23, 2016.

Published online: September 26, 2016. 